\title{
Characteristics of Patients with Pseudoexfoliation Syndrome at a Tertiary Eye Care Center in Jordan: A Retrospective Chart Review
}

\author{
Hisham Jammal (D) - Mohammed Abu Ameera • Noor Al Qudah • \\ Mohammed Aldalaykeh • Areen Abukahel - Ahmed Al Amer • \\ Muawyah Al Bdour
}

Received: September 26, 2020 / Accepted: October 23, 2020 / Published online: October 29, 2020

(C) The Author(s) 2020

\section{ABSTRACT}

Purpose: To present the clinical profile of patients with pseudoexfoliation (PXF) and describe its association with pseudoexfoliation glaucoma and cataract surgery complications at a university hospital in Jordan.

Methods: Electronic chart search was performed among patients aged $\geq 50$ years who attended the Jordan University Hospital between January 2015 and March 2018, to identify patients with pseudoexfoliation. Data derived from history and ophthalmic examination regarding laterality of pseudoexfoliation, presence of glaucoma, glaucoma surgery, cataract, cataract surgery, lens instability, and

H. Jammal ( $₫) \cdot$ N. Al Qudah

Department of Ophthalmology, Faculty of

Medicine, Jordan University of Science and

Technology, Irbid, Jordan

e-mail: hmjammal@just.edu.jo

M. Abu Ameera · M. Al Bdour

Department of Ophthalmology, Faculty of

Medicine, The University of Jordan, Amman, Jordan

M. Aldalaykeh

Community and Mental Health Nursing

Department, College of Nursing, Jordan University

of Science and Technology, Irbid, Jordan

A. Abukahel · A. Al Amer

Department of Ophthalmology, Jordan University

Hospital, Amman, Jordan complications of cataract surgery were collected and analyzed.

Results: Of 19,753 patient records searched, 962 (477 male and 485 female) had PXF (4.9\%). The mean (SD) age was 71.8 (8.1) years. Pseudoexfoliation was unilateral in 539 patients $(56.0 \%)$. The mean age of patients with clinically bilateral PXF was significantly higher than those with clinically unilateral PXF $(p=0.001)$. Pseudoexfoliation glaucoma was present in 237 of 962 (25.4\%) patients with PXF and was significantly associated with clinically bilateral PXF $(p<0.001)$ and male gender $(p=0.001)$. In 454 (48.9\%) patients (638 eyes) who underwent cataract surgery, there was no statistically significant difference in the rate of intraoperative complications between PXF eyes and the fellow eyes of clinically unilateral PXF eyes $(p=0.37)$, or between patients with clinically unilateral and clinically bilateral PXF $(p=0.78)$.

Conclusion: In this large hospital-based study, the frequency of PXF was 4.9\%. Glaucoma was present in one fourth of patients and was significantly associated with clinically bilateral PXF and male gender. Cataract surgery complication rates were not statistically different between PXF and the fellow eyes of clinically unilateral PXF eyes. Surgeons need to be aware of the potential increased risk of intraoperative complications in both groups.

Keywords: Cataract surgery; Complications; Glaucoma; Jordan; Pseudoexfoliation 


\section{Key Summary Points}

\section{Why carry out this study?}

Pseudoexfoliation syndrome is the most common cause of secondary open-angle glaucoma and is associated with increased risk of cataract surgery complications.

Pseudoexfoliation syndrome is known to be a bilateral disease despite the presence of clinically apparent unilateral cases.

This study aimed to assess the frequency of pseudoexfoliation in a large number of patients attending a university teaching hospital and to describe its association with glaucoma and cataract surgery complications.

\section{What was learned from the study?}

Glaucoma was present in nearly one fourth of patients with pseudoexfoliation. Cataract surgery complications did not differ between eyes with pseudoexfoliation and clinically no pseudoexfoliation.

Surgeons need to be aware of the potentially increased risk of intraoperative complications in eyes with pseudoexfoliation as well as fellow eyes clinically labeled as normal eyes in patients with clinically unilateral pseudoexfoliation.

\section{DIGITAL FEATURES}

This article is published with digital features, including a summary slide, to facilitate understanding of the article. To view digital features for this article go to https://doi.org/10.6084/ m9.figshare.13129772.

\section{INTRODUCTION}

Pseudoexfoliation (PXF) syndrome is a disease in which extracellular fibrillar material is deposited on the surface of many ocular structures and in the connective tissues of many visceral organs. This material is composed of elastic fiber components, basement membrane and extracellular matrix components, and blood-derived and immune-related proteins $[1,2]$. The disease is associated with lysyl oxidase-like-1 (LOXL1) gene abnormality [3] with resultant abnormal chemical and mechanical properties of elastic tissues such as the lens zonules, trabecular meshwork, and lamina cribrosa [2]. Other epigenetic and environmental factors may be involved as well [4].

In the eye, pseudoexfoliation material (PXM) accumulates on the lens surface, iris, ciliary body, zonules, corneal endothelium, and trabecular meshwork. In addition, pupillary ruff atrophy and increased trabecular meshwork pigmentation are present. Clinically, the appearance of white dandruff-like material associated with iris transillumination defects, inadequate pupil dilation, lens instability, and corneal endothelial abnormalities are well-recognized features, but the high risk of developing pseudoexfoliation glaucoma (PXG) [5] and the increased risk of cataract surgery complications are the most important clinical implications of the disease.

Overall, PXF is the most common identifiable cause of open-angle glaucoma and is usually associated with higher intraocular pressure (IOP) levels [6]. Although not all patients with PXF will develop glaucoma, a significant percentage will develop glaucoma, which is thought to be more difficult to manage and has higher treatment failure than primary openangle glaucoma [7]. The risk of developing glaucoma in PXF is three times that for patients without PXF for the same high IOP (above $21 \mathrm{mmHg}$ ) [8].

PXF is also associated with an increased risk of cataract, cataract surgery [9], and cataract surgery complications, including intraoperative vitreous loss, postoperative IOP spikes, capsular phimosis, inflammation, and corneal 
decompensation [10]. The cataract in PXF is commonly dense nuclear [11] and frequently coexists with glaucoma, sometimes necessitating combined cataract and glaucoma surgery.

The prevalence of PXF differs widely among populations [12]; however, the highest rates have been reported from Scandinavian countries, Greece [13], and sub-Saharan Africa [14], while PXF tends to occur much less frequently in Asian populations [15]. In the Middle East, rates ranging from 3.5 to $9.1 \%$ in hospital-based studies [16-18] have been reported. The differences in prevalence rates probably reflect the complexity of all factors involved in the pathogenesis of PXF, apart from differences in study design and examiners' ability to detect early cases.

In this study, we present the clinical profile of 962 patients with pseudoexfoliation syndrome and describe the association with glaucoma and cataract surgery complications in a teaching university hospital in Jordan.

\section{METHODS}

\section{Study Design and Population}

The study was a retrospective electronic chart review of patients with pseudoexfoliation syndrome. We conducted a search in the electronic medical records database system at the Jordan University hospital (Amman, Jordan) to identify all new and consecutive patients aged 50 years or older with pseudoexfoliation syndrome who attended the eye outpatient clinic between January 1, 2015, and March 30, 2018. The study was conducted according to the principles of the Declaration of Helsinki. Ethical approval was obtained from the Institutional Review Board at the Jordan University of Science and Technology (Approval number 20200251).

\section{Study Instruments}

To find patients with pseudoexfoliation, we entered the following search terms: pseudoexfoliation, pseudoexfoliation glaucoma, PXF, PEX, and PXG.

All available demographic and clinical data for each patient with pseudoexfoliation were retrieved and entered on a spreadsheet. Demographic data included age and gender. Clinical data derived from history and ophthalmic examination regarding the presence of diabetes mellitus (DM), laterality of pseudoexfoliation, glaucoma, ocular hypertension (OHT), glaucoma surgery, cataract, cataract surgery, signs of lens instability, and complications of cataract surgery were also collected. Patients with ambiguous diagnoses or significantly incomplete data regarding important diagnostic entities were excluded.

Pseudoexfoliation was considered present when typical PXM was present at the pupil, lens surface, or other intraocular structures. When no PXM was seen in one eye after pupil dilation, the eye was considered clinically non-PXF, and the case labeled as clinically unilateral PXF.

Pseudoexfoliation glaucoma (PXG) was defined as the presence of PXF and clinical glaucomatous optic neuropathy manifested as focal or diffuse neuroretinal rim thinning, retinal nerve fiber layer defects, or peripapillary atrophy, with corresponding glaucomatous visual field defects manifested as nasal step, temporal wedge defect, classic arcuate defect, paracentral defect, generalized visual field constriction, or retinal nerve fiber layer defects on optical coherence tomography (OCT). PXF patients with prior surgical intervention for glaucoma or currently receiving glaucoma topical medications and meeting the above definition of glaucoma were considered PXG cases. Ocular hypertension was defined as intraocular pressure above $21 \mathrm{mmHg}$ with no evidence of glaucomatous optic nerve damage, glaucomatous visual field abnormality, retinal nerve fiber layer abnormality on OCT, and the absence of secondary causes of high intraocular pressure.

Cataract was defined as the presence of any degree of lens opacity. Cataract surgery was performed by a consultant or senior resident under consultant supervision. When the outcome of cataract surgery was a sulcus intraocular lens (IOL), anterior chamber IOL, or aphakia, 
as documented in the operative notes, cataract surgery was considered complicated.

\section{Data Analysis}

Data were analyzed using IBM SPSS version 24 software. We used mean (standard deviation) to describe continuous variables and used count (percentage) to describe categorical variables. We performed independent-samples $t$ tests to compare mean age between groups. The chisquare $\left(\chi^{2}\right)$ test was used to analyze the difference between categorical variables. Logistic regression was used to analyze factors associated with glaucoma and cataract surgery complications. A $p$ value of 0.05 or less was considered statistically significant.

\section{RESULTS}

\section{Patient Characteristics}

The electronic medical records (EMR) search included 19,753 patients aged 50 years or older. A total of $962(4.9 \%)$ patients were found to have pseudoexfoliation syndrome and were included in the study. The mean (SD) age of the 962 patients was 71.8 (8.1) years (range $50-95$ years). Nearly half of the patients (46.9\%) were aged 70-79 years. Age and gender distribution are shown in Table 1 . There was no statistically significant mean age difference between men and women $(t=1.42, p=0.162)$. Three hundred and fifty-seven patients (37.1\%) had DM. PXF was clinically unilateral in 539 (56.0\%) patients, and clinically bilateral in 423 (44.0\%) patients. Of the patients with clinically unilateral PXF, 17 patients had lost the other eye due to either trauma or previous complicated intraocular surgery (total eyes: 1385 PXF eyes, 522 clinically non-PXF eyes). The mean age of patients with clinically bilateral PXF (72.8) was significantly higher than those with clinically unilateral PXF (71.0) $(t=-3.351$, $p=0.001$ ), but no gender difference was found between clinically unilateral and clinically bilateral cases $\quad\left(\chi^{2}=1.447, \quad p=0.229\right)$.
Table 1 Age and gender distribution of 962 patients with PXF

\begin{tabular}{llr}
\hline & Number & $\%$ \\
\hline Gender & 477 & \\
Male & 485 & 49.6 \\
Female & & 50.4 \\
Age (years) & 72 & 7.5 \\
$50-59$ & 282 & 29.3 \\
$60-69$ & 451 & 46.9 \\
$70-79$ & 157 & 16.3 \\
$80+$ & 962 & 100.0 \\
Total & &
\end{tabular}

Combined cataract and glaucoma were present in $248(25.8 \%)$ patients.

\section{Glaucoma}

Overall, glaucoma was present in 253 (26.3\%) patients and was bilateral in $184(72.7 \%)$ of the glaucoma cases. Open-angle glaucoma was the most common type, occurring in 243 patients (96.0\%). Of all glaucoma patients, 230 (90.9\%) patients were medically controlled, and 23 patients underwent surgery to control IOP. Table 2 shows the characteristics of glaucoma and glaucoma procedures performed.

After excluding patients with secondary glaucoma (neovascular and post-complicated intraocular surgery), patients with lost eyes, and patients with OHT, 932 patients with PXF remained (a total of 1864 eyes: 838 eyes of 419 clinically bilateral PXF patients, and 513 PXF eyes and 513 clinically non-PXF eyes of 513 patients with clinically unilateral PXF). Pseudoexfoliation glaucoma (PXG) was present in 237 of 962 (25.4\%) patients with PXF, of whom $55(23.2 \%)$ had unilateral glaucoma, and 182 (76.8\%) had bilateral glaucoma. There was a significant gender difference in the frequency of glaucoma $\left(\chi^{2}=10.963, \quad p=0.001\right) ; \quad$ men $(n=140,30.2 \%)$ had a higher frequency of 
Table 2 Characteristics of glaucoma and glaucoma procedures performed

\begin{tabular}{ll}
\hline Variable & Number (\%) \\
\hline Presence of glaucoma & Glaucoma $253(26.3 \%)$, ocular hypertension $7(0.7 \%)$, normal $702(73.0 \%)$ \\
Glaucoma side & Unilateral $69(27.3 \%)$, bilateral $184(72.7 \%)$ \\
Glaucoma type & Open-angle 243 (96\%), secondary $7(2.8 \%)$, angle-closure $3(1.2 \%)$ \\
Glaucoma procedures & Bilateral trabeculectomy 8, unilateral trabeculectomy 10, unilateral Ahmed valve 5 \\
\hline
\end{tabular}

glaucoma than women $(n=97,20.7 \%)$. The frequency of glaucoma was also significantly different between clinically bilateral PXF cases $(n=139,33.2 \%)$ and clinically unilateral PXF cases $(n=98,19.1 \%)\left(\chi^{2}=24.080 p<0.001\right)$. Bilateral glaucoma was more common in clinically bilateral PXF ( $n=133,95.7 \%)$ compared to clinically unilateral PXF $(n=49, \quad 50 \%)$ $\left(\chi^{2}=67.309 p<0.001\right)$. No significant differences were found in relation to the presence of $\mathrm{DM}\left(\chi^{2}=2.069, p=0.150\right)$ or to the mean age between patients with or without glaucoma $(t=1.278, p=0.201)$.

We ran binary logistic regression using four predictors (age, gender, DM status, and laterality of PXF) to predict glaucoma status. The whole model was significant $\left(\chi^{2}(4)=39.848\right.$, $p<0.001)$. The model also showed a good fit as evidenced by no significance of the Hosmer-Lemeshow test $\left(\chi^{2}(8)=14.317, p=0.074\right)$. Age, gender, and laterality were significantly associated with glaucoma among patients with pseudoexfoliation. Patients with clinically bilateral PXF were 2.149 times as likely to have glaucoma (odds ratio [OR] 2.15) as those with clinically unilateral PXF. Male gender was also associated with higher odds of glaucoma (OR 1.649), whereas increased age was less associated with glaucoma (OR 0.980). Table 3 shows the results of logistic regression analysis for the prediction of glaucoma.

\section{Cataract and Surgical Complications}

Cataract was present in 928 (96.5\%) patients, of whom $454(48.9 \%)$ underwent cataract extraction in either one or both eyes (unilateral/bilateral: 270:184 patients). Of the total 638 eyes
Table 3 Binary logistic regression for prediction of glaucoma

\begin{tabular}{ccccc}
\hline Variable & OR & \multicolumn{3}{l}{ 95\% CI for OR } \\
\cline { 3 - 5 } & & Lower & Upper & $\boldsymbol{p}$ \\
\hline Bilateral & 2.149 & 1.583 & 2.916 & 0.000 \\
$\quad$ PXF & & & & \\
Male gender & 1.649 & 1.216 & 2.235 & 0.001 \\
DM & 0.814 & 0.592 & 1.119 & 0.205 \\
Age (years) & 0.980 & 0.962 & 0.998 & 0.033 \\
\hline
\end{tabular}

$C I$ confidence interval, $O R$ odds ratio, $P X F$ pseudoexfoliation, $D M$ diabetes mellitus

that underwent surgery, PXF was present in 499 (78.2\%) eyes. Of all PXF study patients, 50 (5.2\%) patients had iridodonesis or phacodonesis in either one or both eyes. Sixty-one of $499(12.2 \%)$ eyes with PXF had cataract surgery complications, while 21 of 139 (15.1\%) clinically non-PXF eyes developed complications. Table 4 shows the distribution of cataract surgery complications among eyes with PXF and fellow eyes of clinically unilateral PXF. The difference in the frequency of complications between the two groups was not significant $\left(\chi^{2}\right.$ $(1)=0.807, p=0.37)$. There was also no difference in complication rate between patients with clinically unilateral pseudoexfoliation $(n=37$, $15.6 \%)$ and clinically bilateral pseudoexfoliation $(n=36,16.6 \%)\left(\chi^{2}(1)=0.08, p=0.78\right)$.

In binary logistic regression, age, laterality of PXF, DM status, gender, and lens instability were entered in the model to predict cataract surgery complications. The whole model was significant $\left(\chi^{2} \quad(5)=21.54, \quad p<0.01\right), \quad$ and 
Table 4 The distribution of cataract surgery complications among eyes with PXF and fellow eyes of clinically unilateral PXF

\begin{tabular}{llllll}
\hline & \multicolumn{3}{l}{ Complicated cataract surgery $\boldsymbol{n}, \boldsymbol{\%}$} & \multirow{2}{*}{ Total } \\
\cline { 2 - 5 } & None & AC IOL & Sulcus IOL & Aphakia & \\
\hline Fellow eyes of clinically unilateral PXF & 118 & 11 & 7 & 3 & 139 \\
& $84.9 \%$ & $7.9 \%$ & $5.0 \%$ & $2.2 \%$ & $100.0 \%$ \\
PXF eyes & 438 & 24 & 25 & 12 & 499 \\
\multirow{2}{*}{ Total } & $87.8 \%$ & $4.8 \%$ & $5.0 \%$ & $2.4 \%$ & $100.0 \%$ \\
& 556 & 35 & 32 & 15 & 638 \\
& $87.1 \%$ & $5.5 \%$ & $5.0 \%$ & $2.4 \%$ & $100.0 \%$ \\
\hline
\end{tabular}

PXF pseudoexfoliation, $A C$ anterior chamber, $I O L$ intraocular lens

Hosmer-Lemeshow goodness of fit was not significant $\left(\chi^{2}(8)=7.42, p=0.492\right)$. Gender and age were the only variables with a significant effect on predicting cataract surgery complications. Male gender was associated with lower odds of cataract surgery complications (OR 0.494), and increased age was associated with a greater likelihood of cataract surgery complications (OR 1.060). Table 5 shows the results of logistic regression analysis for the prediction of cataract surgery complications.

\section{DISCUSSION}

This study, which included 19,753 patients, is the largest study to date conducted on a hospital-based population of consecutive patients in Jordan for the purpose of detecting patients with PXF. The frequency of PXF was $4.9 \%$. Compared with the results of a previous hospital-based study conducted in 2008 at the same hospital [18] and involving 1195 consecutive patients, this study revealed a lower frequency of PXF (4.9\% vs. 9.1\%). Both studies showed no gender difference, but in the present study, cases of clinically unilateral PXF were reported more frequently than clinically bilateral cases. The frequency of glaucoma was slightly lower in this study (25.4\% vs. $33.1 \%)$.

A great deal of debate exists regarding differences in prevalence rates of PXF among different populations, and these rates vary even within the same country [12]. Most of these differences exist because of either true ethnic differences, or different sex/age distribution of

Table 5 Binary logistic regression for prediction of cataract surgery complications

\begin{tabular}{lllll}
\hline Variable & OR & \multicolumn{2}{l}{$\mathbf{9 5 \%}$ CI for OR } & \multirow{2}{*}{\begin{tabular}{ll} 
Opper \\
\cline { 3 - 4 }
\end{tabular}} \\
\cline { 3 - 4 } Age (years) & 1.060 & 1.024 & 1.097 & 0.001 \\
Laterality of PXF & 1.025 & 0.611 & 1.721 & 0.925 \\
DM & 0.617 & 0.353 & 1.081 & 0.091 \\
Male gender & 0.494 & 0.292 & 0.836 & 0.009 \\
Iridodonesis/phacodonesis & 1.137 & 0.485 & 2.667 & 0.768 \\
\hline
\end{tabular}

$C I$ confidence interval, $O R$ odds ratio, $P X F$ pseudoexfoliation, $D M$ diabetes mellitus 
the studied population, in addition to different inclusion criteria, definition of early PXF cases, and the examiners' ability to detect cases with subtle signs of PXF [19]. Therefore, the lower frequency rate reported in the current study may not represent a true variance from the previous study by Al Bdour et al. [18]. However, the true frequency of PXF may be significantly higher than what we reported, for many reasons. It is possible that many pseudophakic patients were not included in the study, as PXM may be difficult to see in those patients [20]. The presence of cataract, which is commonly associated with PXF and a common presenting diagnosis among the study population, may also hinder the detection of PXM on the lens surface. Failure to re-examine the lens with a slit lamp after dilating the pupil is another factor. Furthermore, inaccurate data entry and misspelling of diagnostic terminology will exclude many cases.

Regarding age, most patients with PXF were in the age category of 70-79 years, and this is consistent with the fact that PXF is an age-related condition. Published studies on PXF are inconsistent regarding gender differences among PXF patients, and in the present study, we observed no such difference.

Regardless of laterality, PXF is considered a bilateral condition with variable and marked clinical asymmetry [21], and the difference in rates of unilateral or bilateral involvement is most likely due to the examiners' ability to detect early cases, especially because typical PXM may be easily missed in the presence of poor pupil dilation that is characteristic of PXF. In this study, clinically unilateral cases were more prevalent than clinically bilateral cases, and more careful examination may have uncovered missed signs of PXF in the eye labeled as clinically non-PXF. Even in the apparent absence of clinical PXF in one eye, ultrastructural and biomicroscopic changes have been documented in such eyes [21]. There is no agreement among different studies as to which is more common in terms of laterality at point observation, but clinically unilateral PXF converts to clinically bilateral PXF over time and with variable probability [22], hence the slightly older mean age of patients with clinically bilateral PXF in the present study population.

In the present study, $25.4 \%$ of patients with PXF had pseudoexfoliation glaucoma, a higher rate than most studies have reported. Any comparison of glaucoma rates between different studies will suffer from variability in the study design and population involved, the definition of glaucoma, the inclusion of patients with high intraocular pressure only, and the overall prevalence of glaucoma among different ethnicities. It is worth noting that populationbased studies have reported lower rates of glaucoma (2.8-15.2\%) [4] than hospital-based studies, and the argument for this is similar to that for lower PXF rates in population-based studies, as study cohorts attending a hospital are more likely to manifest PXF and glaucoma. However, in the Middle East, we could find only two studies reporting on glaucoma prevalence among PXF patients using the same definition of glaucoma. In Egypt, the prevalence of PXF glaucoma was $30.3 \%$ [17], and previously in Jordan the rate was $33.1 \%$ [18].

PXF glaucoma tends to be more unilateral on presentation [23, 24], yet we found more bilateral cases. However, with time, there is an increased risk of conversion to glaucoma in PXF eyes, and in the clinically normal eye in patients with clinically unilateral PXF [23, 25].

The association between PXF and the future development of glaucoma is well established, although not all patients with PXF will develop glaucoma. In the present study, patients with clinically bilateral PXF were more than twice as likely to have glaucoma (unilateral or bilateral) as those with clinically unilateral PXF; a similar finding was already described by Kozart and Yanoff [26]. We also noted that bilateral glaucoma was more frequent among patients with clinically bilateral PXF, and unilateral glaucoma was more frequent among those with clinically unilateral PXF. Since the conversion from clinically unilateral to clinically bilateral PXF increases with time, it is expected that glaucoma conversion from unilateral to bilateral follows a similar pattern. However, the temporal interaction between the presence of PXF and the development of glaucoma is very complicated, and various mechanisms and 
observations need to be considered when trying to explain this interaction, including age, timerelated deposition of PXM in the trabecular meshwork, pigment liberation, initial high IOP, and other genetic predisposing factors that may manifest with time.

Although previous studies have found PXF glaucoma to be more commonly associated with female gender $[24,27]$, many other studies have found no gender difference in glaucoma [22]. This variation in gender susceptibility has not yet been explained. In the present study, male gender was independently associated with glaucoma.

Phacodonesis with or without iridodonesis is a sign of zonular weakness, which results from degenerative changes in the zonular fibers and impaired fiber anchorage to the lens capsule and ciliary processes [22]. Its presence among PXF patients is variable but appears to be between 2.6 and $10.6 \%$ [28, 29]. In the present study population, $5.2 \%$ of patients had phacodonesis and/or iridodonesis, but the true numbers could be higher, as phacodonesis may be subtle in early cases and especially after pupil dilation.

With pseudoexfoliation, there is an increased risk of developing intraoperative and postoperative cataract surgery complications. Although the risk of these complications has been significantly reduced with the adoption of advanced microsurgical techniques and special instrumentation, the risk remains higher than with normal eyes undergoing cataract extraction [30]. The difficulty in obtaining adequate pupil dilation, the presence of zonular weakness, and the usual presence of harder nuclear cataracts makes surgery more challenging and increases the risk of intraoperative complications such as posterior capsule rupture, vitreous prolapse, and IOL decentration. Postoperatively, there is an increased risk of inflammation, corneal endothelial decompensation, capsular phimosis, and IOP spikes, which may further compromise the optic nerve [7]. Careful preoperative evaluation for signs and extent of zonular weakness, the use of pupil-expanding devices (rings, hooks), the use of instruments to support the capsular bag and the weak zonules (capsule tension rings, capsule hooks), and modification of the surgical technique have all contributed to an overall reduction in surgical complications [31].

Overall, there was a high frequency of intraoperative complications in both eyes with PXF and clinically non-PXF eyes. However, we found no significant difference in the frequency of complications between groups. It is now widely established that PXF is a bilateral condition with variable asymmetry, and that the presence of PXM may be a time-related process, where the original pathophysiologic changes have already been established at the molecular and histologic levels in the other clinically normal eye. Sbeity et al. demonstrated bilateral zonular abnormalities, with extensive changes in the zonules of the fellow eye without clinically evident PXM, using ultrasound biomicroscopy, which may assist in preoperative planning [32]. Durukan found higher lens densitometry measurements and higher corneal densitometry values in both the clinically PXF eyes and the clinically non-affected fellow eye of patients with clinically unilateral PXF compared to eyes of healthy subjects, thus suggesting that PXF is a bilateral but asymmetric condition [11]. The findings by Sbeity et al. and Durukan may indicate that fellow clinically non-PXF eyes may have a similar risk of developing complications related to pseudoexfoliation. Also, and in the presence of such microscopic preclinical abnormalities, the surgeon may consider such eyes as low risk, when they are potentially at higher risk, and may not take the necessary precautions to reduce this risk. This may explain the relatively similar frequency of complications among the two groups of eyes in our study. Therefore, surgeons should apply the same preoperative and intraoperative strategies to avoid complications in both eyes of clinically unilateral pseudoexfoliation. The various preoperative, intraoperative, and postoperative issues related to cataract surgery in PXF are discussed in detail by Shingleton et al. [31]. It may also be advisable to offer patients with PXF the option for cataract removal earlier in the course of the disease, to lower the increased complication rate associated with more advanced dense cataracts. This is especially important in our country, where in 
a previous study nearly $20 \%$ of patients who underwent surgery for cataract had dense and mature cataracts [33].

This study has certain limitations. Its retrospective design may have introduced selection bias. Cases with PXF may have been missed during the examination, compared to a prospective design where the examiner will actively search for PXF. The relatively small sample size of cataract surgical cases may have influenced the lack of difference in complication rates between the clinically PXF eyes and the clinically non-PXF eyes. Inaccurate data entry into the EMR system may also exclude certain eligible patients. On the other hand, the study included a large number of patients, originating from the three major geographical regions of Jordan.

\section{CONCLUSION}

In the largest study conducted on a group of patients in Jordan and the Middle East in general, the frequency of PXF was 4.9\%; glaucoma was present in nearly one fourth of patients, and was significantly associated with clinically bilateral PXF cases and male gender. Cataract surgery complications did not differ between PXF and clinically non-PXF fellow eyes. Surgeons therefore need to be aware of the potentially increased risk of intraoperative complications in both groups.

\section{ACKNOWLEDGEMENTS}

Funding. No funding or sponsorship was received for this study or publication of this article. The Rapid Service Fee was funded by the authors.

Authorship. All named authors meet the International Committee of Medical Journal Editors (ICMJE) criteria for authorship for this article, take responsibility for the integrity of the work, and have given their approval for this version to be published.
Disclosures. Hisham Jammal, Mohammed Abu Ameera, Noor Al Qudah, Mohammed Aldalaykeh, Areen Abukahel, Ahmed Al Amer, and Muawyah Al Bdour declare that they have no conflict of interest.

Compliance with Ethics Guidelines. The study was conducted according to the principles of the Declaration of Helsinki. Ethical approval was obtained from the Institutional Review Board at the Jordan University of Science and Technology (Approval number 20200251).

Data Availability. The datasets generated during and/or analyzed during the current study are available from the corresponding author on reasonable request.

Open Access. This article is licensed under a Creative Commons Attribution-NonCommercial 4.0 International License, which permits any non-commercial use, sharing, adaptation, distribution and reproduction in any medium or format, as long as you give appropriate credit to the original author(s) and the source, provide a link to the Creative Commons licence, and indicate if changes were made. The images or other third party material in this article are included in the article's Creative Commons licence, unless indicated otherwise in a credit line to the material. If material is not included in the article's Creative Commons licence and your intended use is not permitted by statutory regulation or exceeds the permitted use, you will need to obtain permission directly from the copyright holder. To view a copy of this licence, visit http:// creativecommons.org/licenses/by-nc/4.0/.

\section{REFERENCES}

1. Ovodenko B, Rostagno A, Neubert TA, Shetty V, Thomas S, Yang A, et al. Proteomic analysis of exfoliation deposits. Invest Ophthalmol Vis Sci. 2007;48:1447-57. https://doi.org/10.1167/iovs.060411.

2. Vazquez LE, Lee RK. Genomic and proteomic pathophysiology of pseudoexfoliation glaucoma. 
Int Ophthalmol Clin. 2014;54:1-13. https://doi. org/10.1097/IIO.0000000000000047.

3. Thorleifsson G, Magnusson KP, Sulem P, Walters GB, Gudbjartsson DF, Stefansson H, et al. Common sequence variants in the LOXL1 gene confer susceptibility to exfoliation glaucoma. Science. 2007;317:1397-400. https://doi.org/10.1126/ science.1146554.

4. Anastasopoulos E, Founti P, Topouzis F. Update on pseudoexfoliation syndrome pathogenesis and associations with intraocular pressure, glaucoma and systemic diseases. Curr Opin Ophthalmol. 2015;26:82-9. https://doi.org/10.1097/ICU. 0000000000000132 .

5. Mitchell P, Wang JJ, Hourihan F. The relationship between glaucoma and pseudoexfoliation: the blue mountains eye study. Arch Ophthalmol. 1999;117: 1319-24. https://doi.org/10.1001/archopht.117.10. 1319.

6. Ritch R, Schlötzer-Schrehardt U, Konstas AGP. Why is glaucoma associated with exfoliation syndrome? Progress iRetinal Eye Res. 2003;22:253-75. https:// doi.org/10.1016/S1350-9462(02)00014-9.

7. Schlötzer-Schrehardt U, Naumann GOH. Ocular and systemic pseudoexfoliation syndrome. Am J Ophthalmol. 2006;141(921-937):e2. https://doi. org/10.1016/j.ajo.2006.01.047.

8. Topouzis F, Harris A, Wilson MR, Koskosas A, Founti P, Yu F, et al. Increased likelihood of glaucoma at the same screening intraocular pressure in subjects with pseudoexfoliation: the Thessaloniki eye study. Am J Ophthalmol. 2009;148(606-613): e1. https://doi.org/10.1016/j.ajo.2009.03.024.

9. Kanthan GL, Mitchell P, Burlutsky G, Rochtchina E, Wang JJ. Pseudoexfoliation syndrome and the longterm incidence of cataract and cataract surgery: the blue mountains eye study. Am J Ophthalmol. 2013;155(83-88):e1. https://doi.org/10.1016/j.ajo. 2012.07.002.

10. Sangal N, Chen TC. Cataract surgery in pseudoexfoliation syndrome. Semin Ophthalmol. 2014;29: 403-8. https://doi.org/10.3109/08820538.2014. 959189.

11. Durukan I. Evaluation of corneal and lens clarity in unilateral pseudoexfoliation syndrome: a densitometric analysis. Clini Exp Optom. 2018;101:740-6. https://doi.org/10.1111/cxo.12802.

12. Konstas AGP, Ringvold A. Epidemiology of exfoliation syndrome. J Glaucoma. 2018;27:S4-11. https://doi.org/10.1097/IJG.0000000000000908.
13. Anastasopoulos E, Topouzis F, Wilson MR, Harris A, Pappas T, Yu F, et al. Characteristics of pseudoexfoliation in the thessaloniki eye study. J Glaucoma. 2011;20:160-6. 0b013e3181d9d8bd.

14. Olawoye OO, Pasquale LR, Ritch R. Exfoliation syndrome in sub-Saharan Africa. Int Ophthalmol. 2014;34:1165-73. https://doi.org/10.1007/s10792014-9953-5.

15. Kim S, Lim SH, Sung KR, Yun SC, Kim CY, Park KH, et al. Prevalence of pseudoexfoliation syndrome and associated factors in South Koreans: The Korean National Health and Nutrition Examination Survey. Ophthalmic Epidemiol. 2016;23:298-302. https://doi.org/10.3109/09286586.2015.1131992.

16. Al-Saleh SA, Al-Dabbagh NM, Al-Shamrani SM, Khan NM, Arfin M, Tariq M, et al. Prevalence of ocular pseudoexfoliation syndrome and associated complications in Riyadh, Saudi Arabia. Saudi Med J. 2015;36:108-12. https://doi.org/10.15537/smj. 2015.1.9121.

17. Shazly TA, Farrag AN, Kamel A, Al-Hussaini AK. Prevalence of pseudoexfoliation syndrome and pseudoexfoliation glaucoma in upper Egypt. BMC Ophthalmol. 2011;11:18. https://doi.org/10.1186/ 1471-2415-11-18.

18. Al-Bdour MD, Al-Till MI, Idrees GM, Abu Samra KM. Pseudoexfoliation syndrome at Jordan University Hospital. Acta Ophthalmol. 2008;86:755-7. https:// doi.org/10.1111/j.1755-3768.2008.01258.x.

19. Ritch R. Exfoliation syndrome. Curr Opin Ophthalmol. 2001;12:124-30.

20. Sternfeld A, Luski M, Sella R, Zahavi A, Geffen N, Pereg A, et al. Diagnosis of pseudoexfoliation syndrome in pseudophakic patients. ORE. 2020. https://doi.org/10.1159/000508336.

21. Hammer T, Schlötzer-Schrehardt U, Naumann $\mathrm{GOH}$. Unilateral or asymmetric pseudoexfoliation syndrome?: an ultrastructural study. Arch Ophthalmol. 2001;119:1023-31. https://doi.org/10. 1001/archopht.119.7.1023.

22. Ritch R, Schlötzer-Schrehardt U. Exfoliation syndrome. Surv Ophthalmol. 2001;45:265-315. https://doi.org/10.1016/S0039-6257(00)00196-X.

23. Jeng SM, Karger RA, Johnson DH, Hodge DO, Burke $\mathrm{J}$, Good MS. Conversion rate of pseudoexfoliation syndrome to pseudoexfoliation glaucoma in a population-based study. Invest Ophthalmol Vis Sci. 2002;43:2947-2947.

24. Musch DC, Shimizu T, Niziol LM, Gillespie BW, Cashwell LF, Lichter PR. Clinical characteristics of 
newly diagnosed primary, pigmentary, and pseudoexfoliative open-angle glaucoma in the collaborative initial glaucoma treatment study. $\mathrm{Br} \mathrm{J}$ Ophthalmol. 2012;96:1180-4. https://doi.org/10. 1136/bjophthalmol-2012-301820.

25. Puska PM. Unilateral exfoliation syndrome: conversion to bilateral exfoliation and to glaucoma: a prospective 10-year follow-up study. J Glaucoma. 2002;11:517-24.

26. Kozart DM, Yanoff M. Intraocular pressure status in 100 consecutive patients with exfoliation syndrome. Ophthalmology. 1982;89:214-8. https:// doi.org/10.1016/S0161-6420(82)34804-6.

27. Kang JH, Loomis S, Wiggs JL, Stein JD, Pasquale LR. Demographic and geographic features of exfoliation glaucoma in 2 United States-Based Prospective Cohorts. Ophthalmology. 2012;119:27-35. https:// doi.org/10.1016/j.ophtha.2011.06.018.

28. Küchle M, Viestenz A, Martus P, Händel A, Jünemann A, Naumann GOH. Anterior chamber depth and complications during cataract surgery in eyes with pseudoexfoliation syndrome. Am J Ophthalmol. 2000;129:281-5. https://doi.org/10.1016/ S0002-9394(99)00365-7.

29. Moreno J, Duch S, Lajara J. Pseudoexfoliation syndrome: clinical factors related to capsular rupture in cataract surgery. Acta Ophthalmol (Copenh). 1993;71:181-4. https://doi.org/10.1111/j.17553768.1993.tb04987.x.

30. Vazquez-Ferreiro P, Carrera-Hueso FJ, Jornet JEP, Fikri-Benbrahim N, Diaz-Rey M, Sanjuan-Cerveró R. Intraoperative complications of phacoemulsification in pseudoexfoliation: metaanalysis. J Cataract Refract Surg. 2016;42:1666-75. https://doi.org/10. 1016/j.jcrs.2016.09.010.

31. Shingleton BJ, Crandall AS, Ahmed IIK. Pseudoexfoliation and the cataract surgeon: preoperative, intraoperative, and postoperative issues related to intraocular pressure, cataract, and intraocular lenses. J Cataract Refract Surg. 2009;35:1101-20. https://doi.org/10.1016/j.jcrs.2009.03.011.

32. Sbeity Z, Dorairaj SK, Reddy S, Tello C, Liebmann JM, Ritch R. Ultrasound biomicroscopy of zonular anatomy in clinically unilateral exfoliation syndrome. Acta Ophthalmol. 2008;86:565-8. https:// doi.org/10.1111/j.1600-0420.2007.01105.x.

33. Jammal HM, Khader Y, Shawer R, Al BM. Posterior segment causes of reduced visual acuity after phacoemulsification in eyes with cataract and obscured fundus view. Clin Ophthalmol. 2012;6:1843-8. https://doi.org/10.2147/OPTH.S38303. 